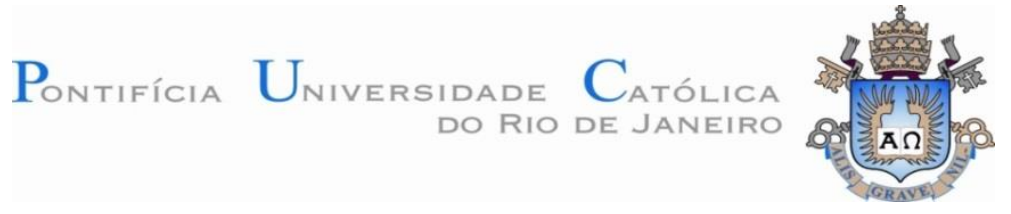

Daniel Peterson Carvalho de Melo

Tecnologia Industrial Básica e Inovação na Base Industrial de Defesa no Brasil

Dissertação apresentada como requisito parcial para obtenção do título de Mestre pelo Programa de Pós-Graduação em Metrologia (Área de concentração: Metrologia para Qualidade e Inovação) da PUC-Rio.

Orientadora: Prof ${ }^{\mathrm{a}}$ Maria Fatima Ludovico de Almeida

Rio de Janeiro

Abril de 2014 


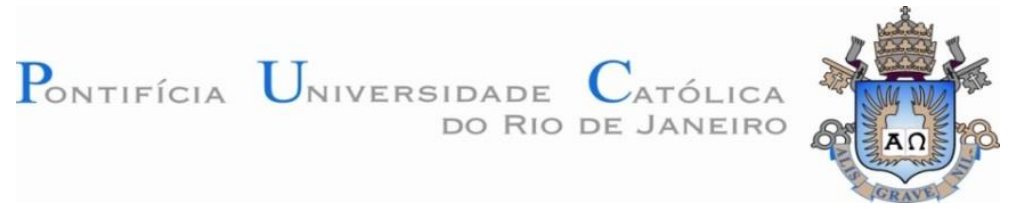

Daniel Peterson Carvalho de Melo

\title{
Tecnologia Industrial Básica e Inovação na Base Industrial de Defesa no Brasil
}

Dissertação apresentada como requisito parcial para obtenção do título de Mestre pelo Programa de Pós-Graduação em Metrologia (Área de concentração: Metrologia para Qualidade e Inovação) da PUC-Rio. Aprovada pela Comissão Examinadora abaixo assinada.

\author{
Prof.- Maria Fatima Ludovico de Almeida \\ Orientadora/Presidente \\ Programa de Pós-Graduação em Metrologia - PUC-Rio
}

Prof. Carlos Augusto Caldas de Moraes

Universidade Cândido Mendes - UCAM

Prof. - Maria Angela Campelo de Melo IAG Escola de Negócios - PUC-Rio

Prof. Maurício Nogueira Frota

Programa de Pós-Graduação em Metrologia - PUC-Rio

Prof. José Eugenio Leal Coordenador Setorial do Centro

Técnico Científico - PUC-Rio

Rio de Janeiro, 25 de abril de 2014 
Todos os direitos reservados. É proibida a reprodução total ou parcial do trabalho sem autorização da universidade, do autor e de sua orientadora.

\section{Daniel Peterson Carvalho de Melo}

Licenciado em Engenharia Elétrica (ênfase Eletrônica) pela Universidade Federal do Rio de Janeiro em 1999. Engenheiro militar do Instituto Militar de Engenharia, desde 1999. Mestrando do Programa de Pós-Graduação em Metrologia para Qualidade e Inovação da PUC-Rio.

Ficha Catalográfica

Melo, Daniel Peterson Carvalho de

Tecnologia industrial básica e inovação na base industrial de defesa no Brasil. / Daniel Peterson Carvalho de Melo; orientadora: Maria Fatima Ludovico de Almeida. - 2014. 148 f. : il. (color.) ; $30 \mathrm{~cm}$

Dissertação (mestrado)-Pontifícia Universidade Católica do Rio de Janeiro, Programa de Pós-Graduação em Metrologia (Área de concentração: Metrologia para Qualidade e Inovação), 2014.

Inclui bibliografia e apêndices

1. Metrologia - Teses. 2. Tecnologia industrial básica. 3 . Inovação. 4. Sistemas setoriais de inovação. 5. Base industrial da defesa. 6. Brasil. 7. PINTEC. I. Almeida, Maria Fatima Ludovico de. II. Pontifícia Universidade Católica do Rio de Janeiro. Programa de Pós-Graduação em Metrologia. III. Título. 


\section{Agradecimentos}

À minha orientadora Professora Maria Fatima Ludovico de Almeida por todo o apoio, ensinamentos e incentivo ao longo de todo o curso de mestrado e pela compreensão, especialmente na fase de elaboração desta dissertação. Para mim, tornou-se uma referência pessoal de profissionalismo e dedicação.

Aos professores do Programa de Pós-graduação em Metrologia para Qualidade e Inovação (PósMQI) e em especial ao Professor Maurício Frota, pela oportunidade de desenvolvimento profissional e acadêmico.

À PUC-Rio e à Coordenação de Aperfeiçoamento de Pessoal de Nível Superior (Capes) pelos auxílios concedidos durante o mestrado.

Ao Instituto Militar de Engenharia e ao Exército Brasileiro, pelo apoio e estímulo durante o desenvolvimento da pesquisa, e por permitir o desenvolvimento profissional de seus engenheiros militares.

À Universidade Federal do Rio de Janeiro, onde iniciei minha vida acadêmica.

Aos colegas de classe pela amizade e companheirismo no decorrer na caminhada deste curso. A todos que me serviram de exemplo, com demonstrações de competência, humildade e respeito.

Aos amigos pelos momentos de descontração e afeto.

À minha família pela compreensão nas minhas horas de ausência.

E a todos que, de alguma forma, contribuíram para a realização deste trabalho. 



\section{Resumo}

Melo, Daniel Peterson Carvalho de. Almeida, Maria Fatima Ludovico de (Orientadora). Tecnologia industrial básica e inovação na base industrial de defesa no Brasil. Rio de Janeiro, 2014. 148 p. Dissertação de Mestrado - Programa de Pós-Graduação em Metrologia (Área de concentração: Metrologia para Qualidade e Inovação), Pontifícia Universidade Católica do Rio de Janeiro.

O objetivo da dissertação é contribuir para o avanço do conhecimento sobre a capacidade de inovação e os obstáculos à inovação enfrentados pelas empresas da Base Industrial da Defesa (BID), destacando-se o papel da Tecnologia Industrial Básica (TIB) no fortalecimento dessa capacidade. Particularmente, a dissertação procura responder como as empresas da BID percebem a importância das funções da TIB - metrologia, normalização, regulamentação técnica e avaliação da conformidade - em seus ciclos de inovação, na perspectiva de ressaltar os benefícios oriundos da consolidação da infraestrutura nacional de serviços tecnológicos de suporte à capacidade inovadora dessas empresas. A metodologia compreende: (i) revisão bibliográfica e documental sobre os temas centrais da dissertação; (ii) definição do objeto do estudo; seleção da fonte primária de dados - Pesquisa de Inovação (Pintec) do IBGE - e da grade de análise da pesquisa; (iii) elaboração do plano tabular para solicitação ao IBGE de tabulações especiais da Pintec 2011; (iv) análise e discussão dos resultados; e (v) formulação das conclusões da pesquisa e de sugestões para a próxima Pintec e estudos futuros. Destacam-se como resultados: (i) a identificação de itens da Pintec diretamente associados às funções da TIB; (ii) a análise da percepção das empresas da BID sobre a relevância das funções da TIB para a inovação; e (iii) a análise comparativa dos padrões de respostas das empresas da BID que implementaram inovações e das que não implementaram.

\section{Palavras-chave}

Metrologia; tecnologia industrial básica; inovação; sistemas setoriais de inovação; base industrial da defesa; Brasil; Pintec. 


\section{Abstract}

Melo, Daniel Peterson Carvalho de. Almeida, Maria Fatima Ludovico de (Advisor). Infrastructural technologies and innovation in the basis of the brazilian industrial defense. Rio de Janeiro, 2014. 148 p. MSc. Dissertation - Programa de Pós-Graduação em Metrologia (Área de concentração: Metrologia para Qualidade e Inovação), Pontifícia Universidade Católica do Rio de Janeiro.

The main aim of this dissertation is to contribute to the understanding of basic infrastructural technologies support to innovation activities by firms of defense industry in Brazil. Particularly, this work seeks to answer how defense firms perceive the importance of infrastructural technologies - metrology, standardization, technical regulation and conformity assessment - in their innovation cycles. The methodology comprises: (i) a literature survey to elaborate a theoretical outline to characterize the core subjects of the research: defense innovation system and defense products; sectorial systems of innovation; and infrastructural technologies; (ii) definition of the research scope; selection of the primary data source - the Brazilian Survey of Technological Innovation (Pintec), published by the Statistical Office (IBGE), and the research analytical framework; (iii) data collection and tabulation; (iv) main findings discussion; and (v) formulation of conclusions and suggestions for the next Pintec survey and for future research. The main results can be summarized as follows: (i) identification of PINTEC items directly associated with basic infrastructural technologies; (ii) identification and analysis of Brazilian defense firms' perceptions about the relevance of basic infrastructural technologies in supporting their innovation strategies; and (iii) comparative analysis of patterns of response of innovative and non-innovative defense firms.

\section{Keywords}

Metrology; infrastructural technologies; innovation; sectorial innovation systems; defense industry; Brazil; Pintec. 


\section{Sumário}

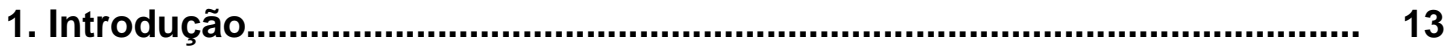

1.1 Definição do problema de pesquisa..................................................... 15

1.2 Objetivos: geral e específicos.................................................................. 16

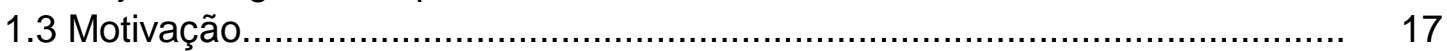

1.4 Metodologia ................................................................................... 18

1.4.1 Fase exploratória e descritiva.......................................................... 18

1.4.2 Pesquisa aplicada...................................................................... 20

1.4.3 Fase conclusiva.................................................................................. 20

1.5 Estrutura da dissertação...................................................................... 21

2. A Base Industrial de Defesa................................................................... 22

2.1 Definição e breve histórico......................................................................... 22

2.2 Panorama atual: características e desempenho da BID............................... 26

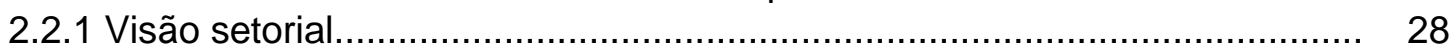

2.2.2 Desempenho competitivo................................................................. 29

2.2.3 Desempenho no comércio exterior...................................................... $\quad 30$

2.3 Políticas e estratégias para o desenvolvimento da BID............................. 32

2.3.1 Estratégia Nacional de Defesa.......................................................... 32

2.3.2 Plano Brasil Maior: um olhar para a BID............................................... 35

2.3.3 Estratégia Nacional de C,T\&I: foco na BID............................................. 36

2.4 Considerações finais sobre o capítulo..................................................... $\quad 37$

\section{Sistemas setoriais de inovação e infraestrutura tecnológica: bases} conceituais.

3.1 Conceitos básicos de sistemas de inovação.............................................. 38

3.2 Componentes centrais de sistemas setoriais de inovação............................. 41

3.2.1 Conhecimento, processo de aprendizado e tecnologias........................... 42

3.2.2 Atores e redes.............................................................................. 43

3.2.3 Instituições................................................................................. 44

3.3 Tecnologia Industrial Básica como fonte de inovação............................... 45

3.3.1 Metrologia.................................................................................. 46

3.3.2 Normalização e regulamentação técnica.............................................. 48

3.3.3 Avaliação da conformidade................................................................. 50

3.3.4 Funções conexas à TIB.................................................................. 52

3.3.4.1 Propriedade intelectual................................................................. 52

3.3.4.2 Informação tecnológica................................................................. 53

3.3.4.3 Tecnologias de gestão..................................................................... 53

3.4 Infraestrutura nacional de serviços tecnológicos....................................... 54

3.5 Considerações finais sobre o capítulo.................................................... 56 
4. Sistema setorial de inovação da defesa no Brasil..................................... 57

4.1 Análise da dinâmica do SSI de defesa................................................... 57

4.1.1 Conhecimento, processo de aprendizado e tecnologias........................... 58

4.1.2 Atores e redes.................................................................................... 59

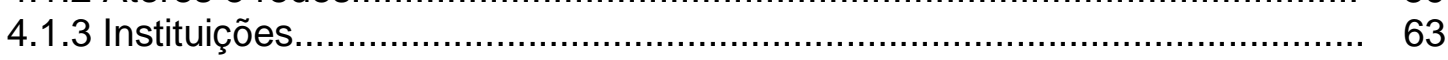

4.2 Novas tecnologias e perspectivas de inovação......................................... 68

4.3 Tecnologia Industrial Básica no SSI de defesa........................................... $\quad 70$

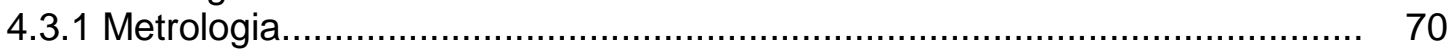

4.3.2 Normalização e regulamentação técnica................................................. 71

4.3.3 Avaliação da conformidade................................................................. 72

4.3.4 Funções conexas à TIB.................................................................... 72

4.4 Considerações finais sobre o capítulo..................................................... 73

5. TIB e fortalecimento da capacidade de inovação das empresas da BID... $\quad 75$

5.1 Definição do objeto de estudo.................................................................... $\quad 75$

5.2 Empresas representativas da Base Industrial de Defesa............................. 76

5.3 Pintec como fonte primária de dados......................................................... 77

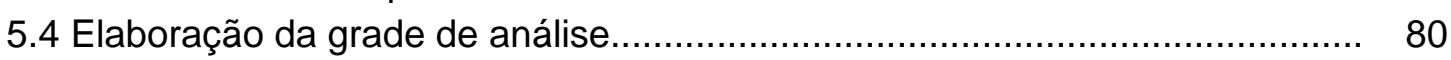

5.4.1 Definição das variáveis........................................................................... 86

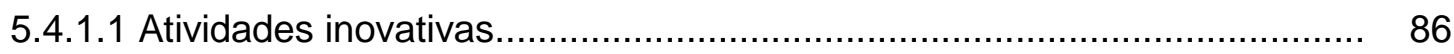

5.4.1.2 Impactos das inovações................................................................ 87

5.4.1.3 Fontes de informação..................................................................... 88

5.4.1.4 Relações de cooperação para inovação............................................. 88

5.4.1.5 Problemas e obstáculos................................................................. 89

5.4.1.6 Inovações organizacionais e de marketing............................................ 89

5.5 Coleta e formatação dos dados................................................................ 90

5.6 Perfil das empresas da BID................................................................... 91

5.7 Resultados referentes a atividades inovativas............................................. 96

5.8 Resultados referentes a impactos das inovações........................................ 99

5.9 Resultados referentes a fontes de informação............................................... 102

5.10 Resultados referentes a relações de cooperação......................................... 105

5.11 Resultados referentes a problemas e obstáculos à inovação........................ 108

5.12 Resultados referentes a inovações organizacionais e de marketing............ 112

5.13 Conclusões do estudo de caso............................................................... 114

6. Conclusões e recomendações............................................................... 118

Referências bibliográficas.................................................................. 123

Apêndice 1 - Plano Tabular para solicitação ao IBGE................................ 132

Apêndice 2 - Tabulação Especial da Pintec 2011 fornecida pelo IBGE......... 138

Apêndice 3 - Relação de empresas associadas à Abimde e selecionadas para a tabulação especial da Pintec 2011.................................. 144 


\section{Lista de Figuras}

Figura 1.1 - Desenho da pesquisa, seus componentes e métodos................. 19

Figura 2.1 - Iceberg científico-tecnológico militar ou BID................................ 23

Figura 2.2 - Evolução dos gastos com defesa no Brasil.................................. 26

Figura 2.3 - Secretaria de Produtos de Defesa (DEPROD) do Ministério da Defesa.......................................................................... 34

Figura 3.1 - Níveis de normalização............................................................ 49

Figura 4.1 - Modelo de análise do SSI de defesa......................................... 57

Figura 4.2 - Organograma resumido do Ministério da Defesa (MD)............... 60

Figura 4.3 - Rede de Cooperação do SisCTID............................................ 66

Figura 5.1 - Estrutura lógica do questionário da Pintec 2011........................ 79

Figura 5.2 - Distribuição percentual das firmas da BID, segundo seus principais ramos de atividade (indústria de transformação e serviços selecionados)........................................................... 92

Figura 5.3 - Distribuição percentual das firmas da BID pertencentes à indústria de transformação......................................................... 93

Figura 5.4 - Distribuição percentual das firmas de serviços da BID, por tipo de serviço selecionado.

Figura 5.5 - Percentual de firmas da BID que implementaram inovações de produto ou processo que atribuíram grau de importância médio ou alto para cada atividade inovativa.

Figura 5.6 - Percentual de firmas da BID que implementaram inovações de produto ou processo que atribuíram grau de importância médio ou alto para cada impacto das inovações.

Figura 5.7 - Percentual de firmas da BID que implementaram inovações de produto ou processo que atribuíram grau de importância médio ou alto para cada fonte de informação.

Figura 5.8 - Percentual de firmas da BID que implementaram inovações de produto ou processo que atribuíram grau de importância médio ou alto para cada relação de cooperação.

Figura 5.9 - Percentual de firmas da BID que implementaram inovações de produto ou processo que atribuíram grau de importância médio ou alto para cada problema ou obstáculo.

Figura 5.10 - Proporção de firmas da BID que implementaram inovações organizacionais ou de marketing. 


\section{Lista de Quadros}

Quadro 3.1 - Fatores e parâmetros para a análise de sistemas setoriais de inovação.

Quadro 4.1 - Segmentos da BID......................................................... 58

Quadro 5.1 - Descrição da grade de análise................................................. 81

\section{Lista de Tabelas}

Tabela 2.1 - Aquisições típicas de defesa (período 2001-2010)

Tabela 2.2 - Quadro de exportações brasileiras de produtos de defesa, a partir de 2000.

Tabela 2.3 -

Maiores exportadores de armamento leve em 2008.

Tabela 5.1 - Número de empresas selecionadas para a Pintec 2011, por atividade e por estrato

Tabela 5.2 - Ramos de atividades das empresas da BID com CNAE

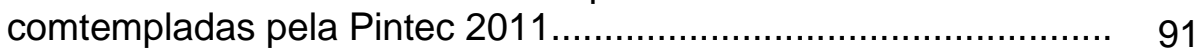

Tabela 5.3 - Taxa de inovação das empresas da BID por estrato: 2000 2011. 


\section{Siglas}

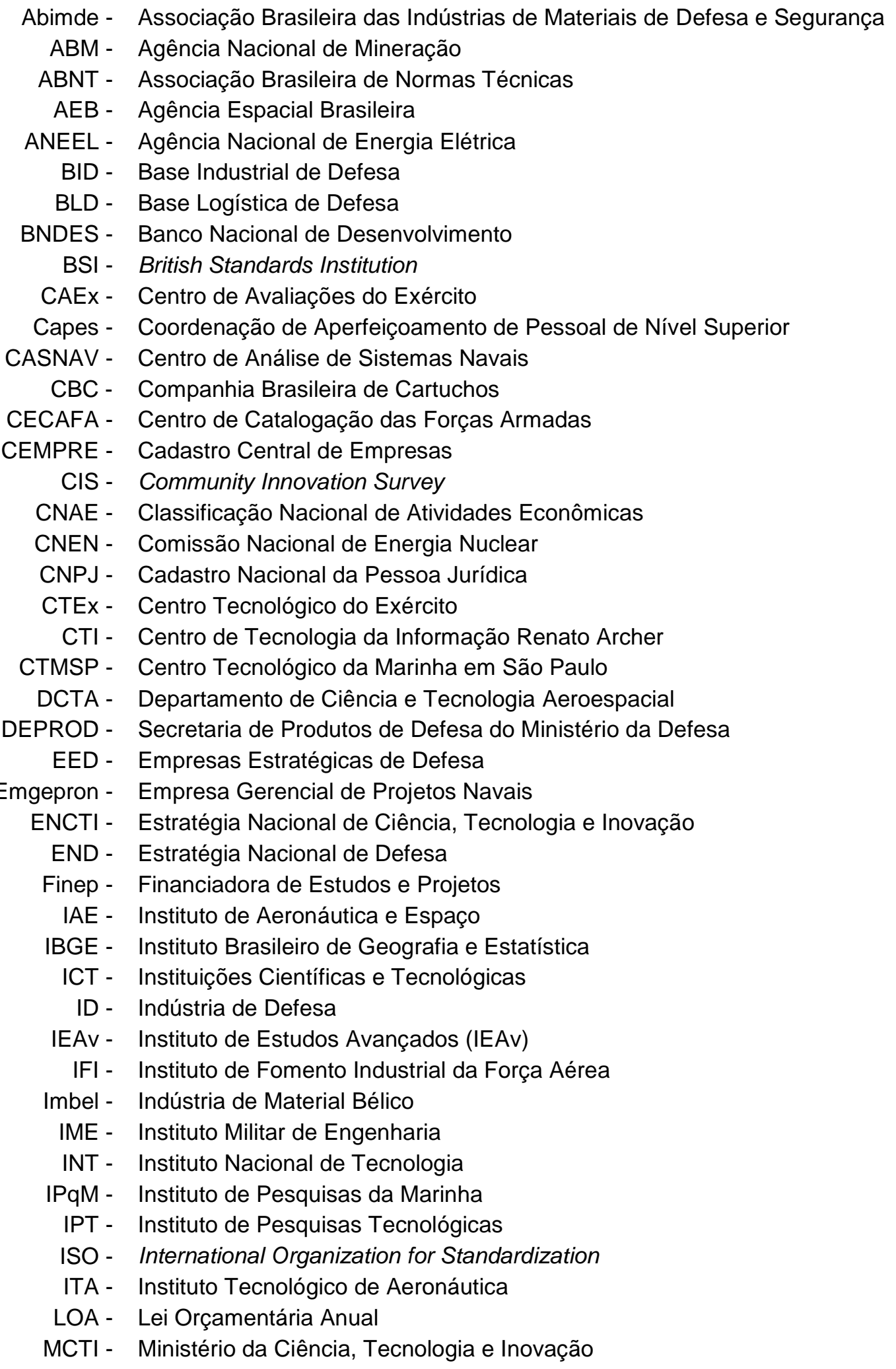


MD - Ministério da Defesa

MDIC - Ministério de Desenvolvimento Industrial e Comércio Exterior

NEE - Núcleo de Estudos Estratégicos da USP

NIT - Núcleo de Inovação Tecnológica

OECD - Organization for Economic Cooperation and Development

OHSAS - Occupational Health \& Safety Advisory Services

$P \& D$ - Pesquisa e Desenvolvimento

PAEB - Plano de Articulação do Exército Brasileiro

PAEMB - Plano de Articulação e Equipamento da Marinha

PAS - Publicly Available Specification

PBM - Plano Brasil Maior

PED - Produtos Estratégicos de Defesa

PEE - Projetos Estratégicos do Exército

PEMAER - Plano Estratégico da Aeronáutica

Pintec - Pesquisa Nacional de Inovação

PNAE - Plano Nacional de Atividades Espaciais

PósMQI - Programa de Pós Graduação em Metrologia da PUC-Rio

PPGEST - Programa de Pós Graduação em Estudos Estratégicos da Defesa e da Segurança da UFF

PRODE - Produto de Defesa

Prosub - Programa Nacional de Desenvolvimento de Submarinos da Marinha

PROTEGER - Sistema Integrado de Proteção de Estruturas Estratégicas Terrestres

PUC-Rio - Pontifícia Universidade Católica do Rio de Janeiro

QUALIESPAÇO - Programa de Apoio às Atividades de Normalização e à Qualidade na Área Espacial

RBC - Rede Brasileira de Calibração

RECOP - Programa de Recuperação da Capacidade Operacional da Força Terrestre

RETID - Regime Especial Tributário para a Indústria de Defesa

SCTA - Sistema de Ciência e Tecnologia da Aeronáutica

SCTEx - Sistema de Ciência e Tecnologia do Exército

SCTM - Sistema de Ciência e Tecnologia da Marinha

SecCTM - Secretaria de Ciência, Tecnologia e Inovação da Marinha

Simde - Sindicato Nacional das Indústrias de Defesa

SINMETRO - Sistema Nacional de Metrologia, Normalização e Qualidade Industrial

SIPLEX - Sistema de Planejamento do Exército

SISCEMEFA - Sistema de Certificação, de Metrologia, de Normalização e Fomento Industrial das Forças Armadas

SisCTID - Sistema de Ciência, Tecnologia e Inovação da Defesa Nacional

SISFRON - Sistema Integrado de Monitoramento de Fronteiras

SISMICAT - Sistema Militar de Catalogação

SMS - Segurança, meio ambiente e saúde ocupacional

SSI - Sistemas setoriais de inovação

TIB - Tecnologia industrial básica

UFF - Universidade Federal Fluminense

UFRJ - Universidade Federal do Rio de Janeiro

USP - Universidade de São Paulo 